\title{
Erratum to: Titles in English-medium Astrophysics research articles
}

\author{
David I. Méndez • M. Ángeles Alcaraz • Françoise Salager-Meyer
}

Published online: 1 March 2015

(C) Akadémiai Kiadó, Budapest, Hungary 2015

\section{Erratum to: Scientometrics (2014) 98:2331-2351 DOI 10.1007/s11192-013-1174-6}

Authors would like to add the following acknowledgement:

Acknowledgments This research was partially supported by Grant No. 1039-13-06-B from the University of the Andes Research Center.

The online version of the original article can be found under doi:10.1007/s11192-013-1174-6.

D. I. Méndez (ه)

Departamento de Física, Ingeniería de Sistemas y Teoría de la Señal, Escuela Politécnica Superior, Universidad de Alicante, Alicante, Spain

e-mail: david.mendez@ua.es

M. Ángeles Alcaraz

Departamento de Estudios Ingleses, Facultad de Filosofía y Letras, Universidad de Alicante, Alicante, Spain

F. Salager-Meyer

Facultad de Medicina, Universidad de los Andes, Mérida, Venezuela 